\title{
Effects of Electrode Spacing and Inversion Techniques on the Efficacy of 2D Resistivity Imaging to Delineate Subsurface Features
}

\author{
Adiat Kola Abdul-Nafiu, Mohd. Nawawi Mohd. Nordin, \\ Khiruddin Abdullah, Ishola Kehinde Saheed and Abdulrahman Abdullah
}

School of Physics, University Sains, Malaysia, 11800 Penang, Malaysia

Received 2012-11-29, Revised 2013-01-31; Accepted 2013-02-27

\begin{abstract}
In this study, the effect of the choice of appropriate electrode spacing and inversion algorithms on the efficacy of 2D imaging to map subsurface features was investigated. The target being investigated was the drainage concrete pipe buried at approximately $0.3 \mathrm{~m}$ into the subsurface. A profile perpendicular to the strike of the pipe was established. 2D resistivity data was separately collected with the electrode spacings of $1.5 \mathrm{~m}$ and $0.5 \mathrm{~m}$. using the Dipole-Dipole, the Wenner and the Wenner-Schlumberger array configurations. The results obtained showed that when the electrode spacing of $1.5 \mathrm{~m}$ was used for the investigations, none of the three array types was able to map the target with either of the two inversion techniques. The results further show that the attainment of RMS error of less about $10 \%$ which usually gives the indication of a good subsurface model is not a guarantee that subsurface features are successfully mapped. On the other hand, when the electrode spacing of $0.5 \mathrm{~m}$ was used for the data collection, the results obtained with the standard constrains inversion technique showed that all the three array configurations mapped the target however, only the dipole-dipole array was able to resolve the boundary between the concrete pipe and the entrapped air. With the robust constrain inversion technique; the target was also successfully mapped by all the three array types. In addition to this, the boundary between the entrapped air and the concrete pipe was resolved by all the three array types. This suggests that if there is a significant contrast in the subsurface layers' resistivities, the robust constrain inversion algorithm technique gives better boundaries resolution irrespective of the array types used for the survey. The inversion of the 3D data gave 3D resistivity sections which were presented as horizontal depth slices. The result obtained from the inversion of the 3D data has assisted us in getting information about the dimension and orientation of the target. The study revealed that a miniature subsurface feature can only be effectively delineated if the feature is not deeply buried into the subsurface.
\end{abstract}

Keywords: 2D, 3D, Subsurface Feature, Target, Standard Constraint, Robust Constraint

\section{INTRODUCTION}

Geophysical methods can be used to examine or delineate subsurface features. This is usually achieved through the observation of the contrasts in the physical properties of such features. Some of the physical properties that are always explored during geophysical investigations include but not limited to density, magnetic susceptibility and electrical resistivity. These physical properties vary between different media involved just as different materials such as clay, concrete; air and water have different geophysical properties. Geophysical surveys provide an efficient way of detecting subsurface heterogeneities such as voids, refilled cavities and the like (Mochales et al., 2008). Several geophysical techniques have been used by different researchers in the past for different forms of subsurface mapping. These techniques include seismic

Corresponding Author: Adiat Kola Abdul-Nafiu, School of Physics, Universiti Sains, Malaysia, 11800 Penang, Malaysia 
refiection and refraction Karl et al. (2011); gravimetry (Rybakov et al., 2001), ground-penetrating radar Friedel et al. (2008) and Sass at al. (2008) and electrical resistivity tomography (Zhou et al., 2002; Santos et al., 2007; Nyari and Kanli, 2007; Santos and Sultan, 2008; Martorana et al., 2009).

In the recent years however, Electrical Resistivity Imaging (ERI) has become one of the most significant geophysical techniques that is commonly used for investigating underground near-surface structures. The electrical resistivity imaging method has been widely used in various engineering, environmental, hydrological, agricultural and mineral surveys (Chambers et al., 2007). This is because the numerous developments in the instrumentation and interpretation techniques have made it possible to carry out 2D and 3D resistivity surveys with maximum time and cost effectiveness.

In this study, we investigated the applicability of ERI survey to the detection of subsurface feature at a location within the main campus of the University Sains, Malaysia. The subsurface feature being investigated was an underground horizontal concrete pipe. Three array types namely the dipole-dipole, the Wenner and the Wenner-Schlumberger were used in the study. The survey was conducted along five parallel lines. The investigation site consists of discrete subsurface structures with sharp boundaries between different bodies. The main objectives of the study were to:

- Establish that the effectiveness of resistivity imaging in delineating subsurface features is largely dependent on the appropriate choice of electrode spacings and inversion algorithms

- Show that the attainment of RMS error of less about $10 \%$ which usually gives the indication of a good subsurface model is not a guarantee that subsurface features are successfully mapped

- Show that the efficacy of resistivity imaging applications in engineering and environmental studies is largely determined by the depth of burial of the subsurface features being investigated

- To obtain the actual geometry of the target through a $3 \mathrm{D}$ resistivity survey

\subsection{Site and Target Descriptions}

The survey site was at the convocation ground of the main campus of the Universiti Sains Malaysia (USM), Pulau Pinang. The topography of the area is gentle. The main campus of USM is characterized by granitic rock; there are several outcrops of the rock in several locations on the campus. The report of the borehole drilled at the vicinity of the survey site shows that the subsurface of the area is made up of two layers which are the top soil and the bedrock. The top soil is made up of a light to dark brown silty clay and some sand and gravel. The composition of sand and gravel varies from fine to coarse. The second layer is the bedrock of granite and this was encountered at a depth of about $42 \mathrm{~m}$ (Alfouzan et al., 2010).

The subsurface feature targeted in this study was an underground horizontal concrete pipe that is being used as a drainage conduit. The pipe has Diameter (D) of 1.61 $\mathrm{m}$ and Thickness $(\mathrm{T})$ of $0.1 \mathrm{~m}$. It was buried at an approximate depth of $0.3 \mathrm{~m}$. The pipe was located along a flood plain.

\section{MATERIALS AND METHODS}

\subsection{Direct Measurement of the Resistivity of the Target and the Surrounding Materials}

Before the data were acquired, the resistivity values of the concrete and the surrounding subsurface materials were determined. Two current cables and two potential cables were clipped to the concrete wall at one end while the other end of the cables was connected to the SAS 4000. The cables were arranged such that constant spacing was maintained between them thus making the arrangement similar to that of Wenner array configuration. The resistance of the concrete was taken for about three times. The resistivity $(\rho)$ of the pipe was obtained by multiplying the value of the average Resistance (R) by the geometric factor $(\mathrm{K})$ of Wenner array (i.e., $\rho=\mathrm{KR}$ ); where $\mathrm{K}=2 \pi \mathrm{a}$ and $\mathrm{a}=$ spacing between the electrodes (cables). The resistivity of the pipe obtained from this process was about $1200 \Omega \mathrm{m}$.

In order to determine the resistivity of the surrounding subsurface materials, four metallic electrodes were buried in to the soil at equal spacing. Using the same process described above, the resistivity of the surrounding subsurface materials was determined to be between 100 and $550 \Omega \mathrm{m}$ (i.e., less than $1000 \Omega \mathrm{m}$ ).

The pipe was filled with air. The resistivity of the air is expected to be higher than that of the concrete just as the resistivity of the concrete was higher than the resistivity of the surrounding subsurface materials. This resistivity contrast will assist in determining the boundaries of the surrounding subsurface materials, the concrete and the entrapped air from the resistivity imaging inversion results. 


\subsection{D Data Acquisition}

A profile was established in a direction perpendicular to the strike of the target as shown. The target, buried at a depth of about $0.3 \mathrm{~m}$ into the subsurface, was located between 7.5 and $9.0 \mathrm{~m}$ from the first electrode position. The data was acquired on the profile using the dipole-dipole electrode array configuration with electrode spacing of $1.5 \mathrm{~m}$. By using the same electrode spacing of $1.5 \mathrm{~m}$, the data acquisition was repeated on the same profile with the Wenner and Wenner-Schlumberger electrode array configurations. The electrode spacing was thereafter changed to $0.5 \mathrm{~m}$ and the process of data acquisition was repeated on the same profile for the three array configurations. The instrument used for the data acquisition was the ABEM Terrameter Signal Averaging System/4000 (Terrameter SAS/4000) and Lund Electrode Selector system. SAS/4000 has the ability to measure in 4 channels at the same time indicating that the measurements can be performed up to four times faster than a mono channel system. Both horizontal and vertical variations in subsurface resistivity were automatically recorded by the instrument.

\subsection{D Data Acquisition}

In addition to the profile used for the $2 \mathrm{D}$ data acquisition, additional four profiles were established parallel to the $2 \mathrm{D}$ profile. The dipole-dipole array was used for the data collection. Measurements were taken along the five profiles. The total length of each profile was $20 \mathrm{~m}$. A spacing of $0.5 \mathrm{~m}$ was used in the $\mathrm{x}$-direction (i.e., electrode spacing) while the spacing in the $y$ direction (i.e., inter-profile spacing) was maintained at 2.0 $\mathrm{m}$. A grid size of $41 \times 5$ was covered during the survey. A total of 205 electrode positions were used. At the end of the survey, the measured data from the five $2 \mathrm{D}$ profiles were collated into one RES3DINV format using the RES2DINV software. A total of 2428 data points was obtained after the collation into RES3DINV format.

\subsection{Inversion of the 2D Data Set}

The collected data resistivity was processed and inverted using the RES2DINV software developed by Loke and Barker (1996). Two inversion techniques were used and these were the standard least-square smoothness constrain and robust constrain inversion techniques.

The standard constrain inversion technique is also called the L2 norm. In this technique, the least-squares method will be used to reduce the square of the differences between the observed and the calculated apparent resistivity values. At the same time, it also attempts to reduce the squares of the changes in the model resistivity values (deGroot-Hedlin and Constable, 1990). This will give a subsurface model whose resistivity values will be smoothly varied. This type of model is suitable in an environment where subsurface resistivity values are changing in a smooth manner (Loke et al., 2003). This method can only produce reasonable results if the data contains random or "Gaussian" noise. If the data set however contains "outlier" data points (i.e., the noise that originates from non-random sources such as mistakes or equipment problems), the results obtained will be less satisfactory. This is because such "outlier" data points could have a great effect on the resulting inversion model.

The robust constrain inversion technique is otherwise called the L1 norm inversion method. The effect of "outlier" data points which affect the resulting inversion model in the L2 norm inversion technique is reduced in the L1 norm method. The L1 norm attempts to reduce the absolute difference between the measured and calculated apparent resistivity values (Loke, 2002). The robust constrain inversion technique produces models with sharp boundaries between different subsurface layers with different resistivity values but with constant resistivity values within each layer. In using the technique, a cut-off factor value must be set. This determines the degree to which the robust data constrain is used. Using a value of 0.05 for instance, implies that the effect of data points where the differences in the measured and calculated apparent resistivity values are much higher than 5 percent will be largely reduced (Loke, 2002)

Generally, the programme automatically creates 2D model by dividing the subsurface into rectangular blocks (Loke and Barker, 1996) and the resistivity of the blocks was iteratively adjusted to reduce the difference between the measured and the calculated apparent resistivity values. The apparent resistivity values were calculated by the finite-difference method. The program calculates the apparent resistivity values and compares these to the measured data. During iteration, the modeled resistivity values will be adjusted until the calculated apparent resistivity values of the model agree with the actual measurements. The iteration is stopped when the inversion process converges (i.e., when the RMS error either falls to acceptable limits, usually less than $5 \%$ or when the change between RMS errors for consecutive iterations becomes infinitesimally small). 
Table 1. A script file is used to combine the 2-D files (after Loke, 2010)

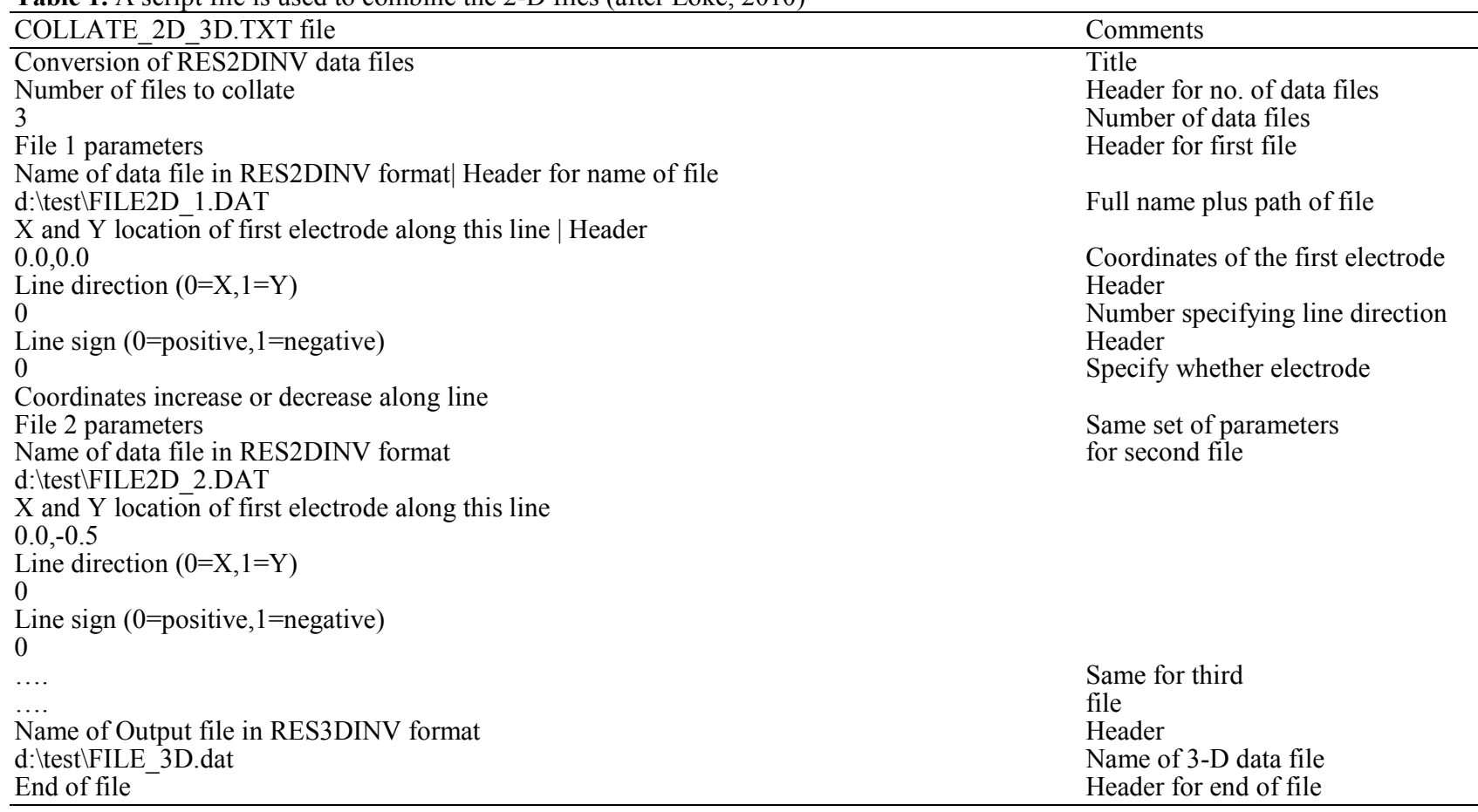

\subsection{Inversion of the 3D Data Set}

In order to carry out $3 \mathrm{D}$ inversion, two steps were taken namely combining $2 \mathrm{D}$ files in to $3 \mathrm{D}$ file format and carrying out the $3 \mathrm{D}$ inversion.

\subsection{Combining 2-D Files in to 3D File Format}

In the RES2DINV program, a number of 2-D data files can be combined into a single 3-D data file. This was done by using the "Collate data into RES3DINV format" option under the "File" menu. This option enables the user the user to combine the 2-D lines with data in the RES2DINV format into a single data file in the format used by the RES3DINV program. A script file is used to combine the 2-D files. The content of this file together with a description of the format used is given in Table 1.

\subsection{D Inversion}

The 3D inversion of the apparent resistivity data will make it possible to obtain the actual geometry of the target. This is important because most of the subsurface features in real world are 3D in nature and these cannot adequately imaged by $2 \mathrm{D}$ survey technique. The robust constrain algorithm inversion technique was used. This was because the result obtained from the $2 \mathrm{D}$ inversion showed that the boundaries were better resolved when the robust constrain algorithm inversion technique was used. The robust constrain cut off was set at 0.1 . Standard Gauss-Newton optimization method was used for the inversion and the convergence limit was set at 0.05 (i.e., $0.5 \%$ accuracy). In order to reduce the number of iterations the program will require to converge, the option to optimize damping factor was chosen. The ratio of the vertical/horizontal filter weight used was 0.5 . The reason for using smaller vertical/horizontal filter weight was because the main anomaly (i.e., the subsurface target) in the apparent resistivity pseudosection is elongated horizontally.

\section{RESULTS}

\subsection{Results of the Standard Constrain Inversion Technique}

The results of the standard constrain inversion technique for the three array configurations with the $1.5 \mathrm{~m}$ electrode spacings are presented as model sections shown in Fig. 1. RMS errors of 4.6, 5.8 and $11.8 \%$ were obtained for the Wenner-Schlumberger, the Wenner and the Dipole-Dipole array configurations respectively. 
Adiat Kola Abdul-Nafiu et al. / American Journal of Applied Sciences, 10 (1): 64-72, 2013

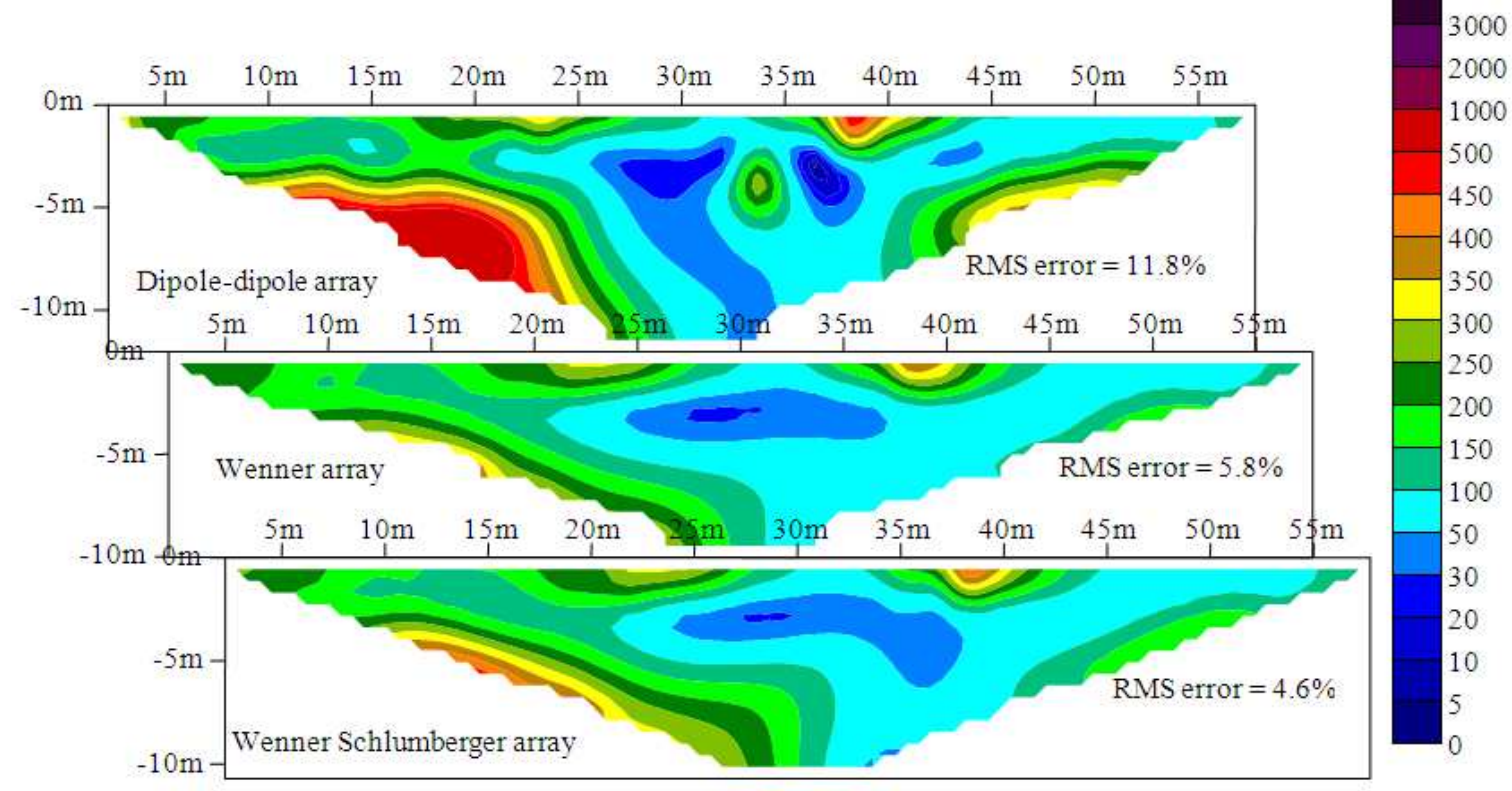

Fig. 1. Inversion results for the three Array Configurations with $1.5 \mathrm{~m}$ electrode spacing using the Standard least-square smoothness constrain inversion technique

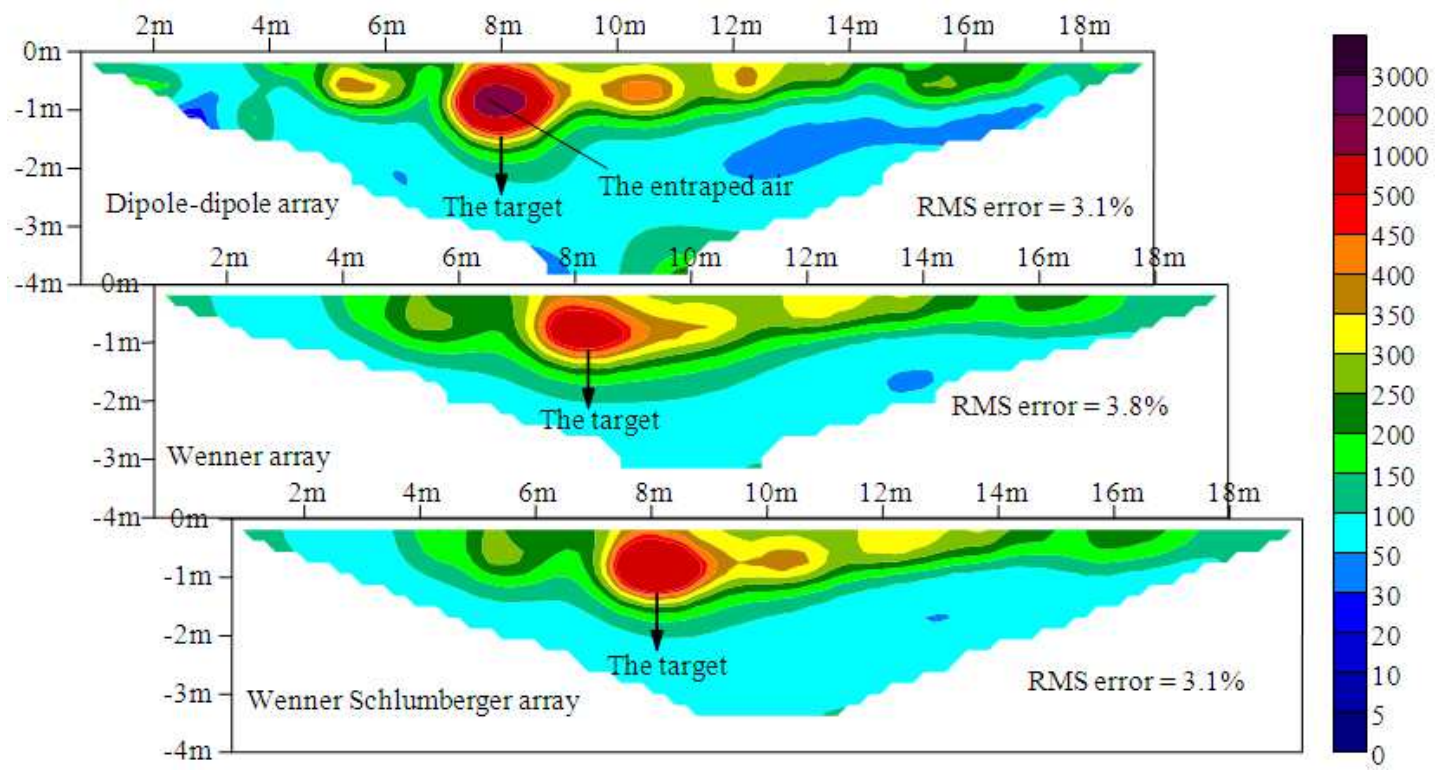

Fig. 2. Inversion results for the three Array Configurations with $0.5 \mathrm{~m}$ electrode spacing using the standard least-square smoothness constrain inversion technique

Similarly, the results of the standard constrain inversion technique for the three array configurations with the $0.5 \mathrm{~m}$ electrode spacings are presented as model sections shown in Fig. 2. RMS errors of 3.1, 3.8 and 3.1\% were obtained for the Wenner-Schlumberger, the Wenner and the Dipole-Dipole array configurations respectively. 


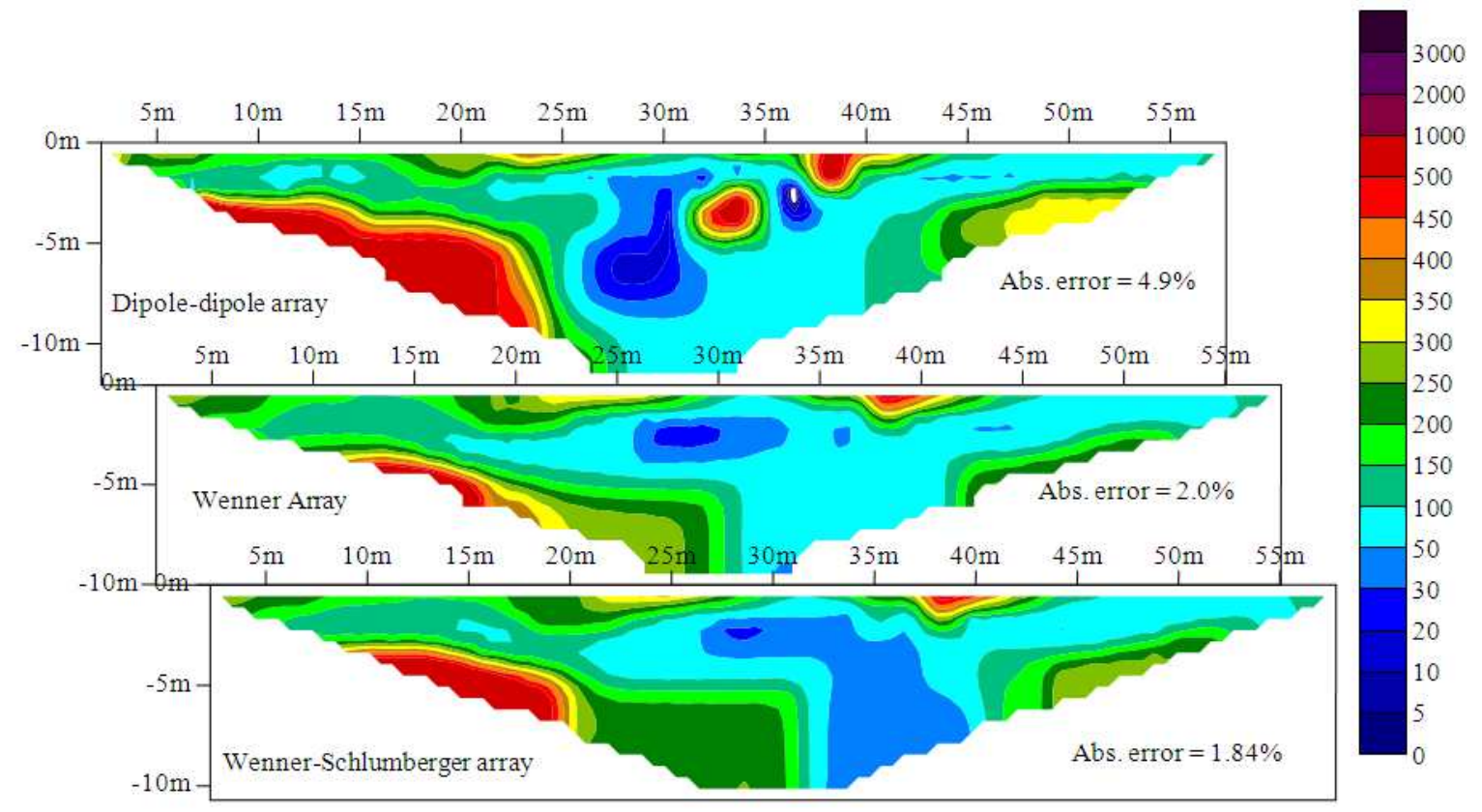

Fig. 3. Inversion Results for the three Array Configurations with $1.5 \mathrm{~m}$ electrode spacing using the Robust constrain inversion technique

\subsection{Results of the Robust Constrain Inversion Technique}

The results of the robust constrain inversion technique for the three array configurations with the $1.5 \mathrm{~m}$ electrode spacings are presented as model sections shown in Fig. 3. Absolute errors of 1.84, 2.0 and 4.9\% were obtained for the Wenner-Schlumberger, the Wenner and the Dipole-Dipole array configurations respectively.

Similarly, the results of the robust constrain inversion technique for the three array configurations with the $0.5 \mathrm{~m}$ electrode spacings are presented as model sections shown in Fig. 4. Absolute errors of 1.14, 1.65 and $2.1 \%$ were obtained for the Wenner-Schlumberger, the Wenner and the Dipole-Dipole array configurations respectively.

\subsection{Results of the Inversion of the 3D Data Set}

The inversion process converged after 5 iterations. The absolute error obtained was $6.14 \%$. The 3D resistivity model is presented as horizontal depth slices shown in Fig. 5. The depth slices show the inverted subsurface resistivity at various depths. The inversion results have assisted in displaying the target in 3 dimensions.

\section{DISCUSSION}

\subsection{Discussions of Results of the Standard Constrain Inversion Technique}

It is revealed from Fig. 1 that none of the three array configuration successfully mapped the target. The inability of any of the three arrays to map the target is because the electrode spacing of $1.5 \mathrm{~m}$ used for the survey is not appropriate for the dimension of the problem at hand (i.e., the target). Though the RMS error of approximately $10 \%$ obtained from all the arrays is an indication of good subsurface models however, the results have shown that obtaining good subsurface model is not an indication that the subsurface target is successfully mapped.

Similarly, it is obvious from Fig. 2 that all the three array configurations successfully mapped the target. However, only the Dipole-Dipole array configuration successfully resolved the entrapped air between the concrete pipes. This suggests that the Dipole-Dipole array configuration gives the best boundaries resolution results among the three array configurations used for the study. 
Adiat Kola Abdul-Nafiu et al. / American Journal of Applied Sciences, 10 (1): 64-72, 2013

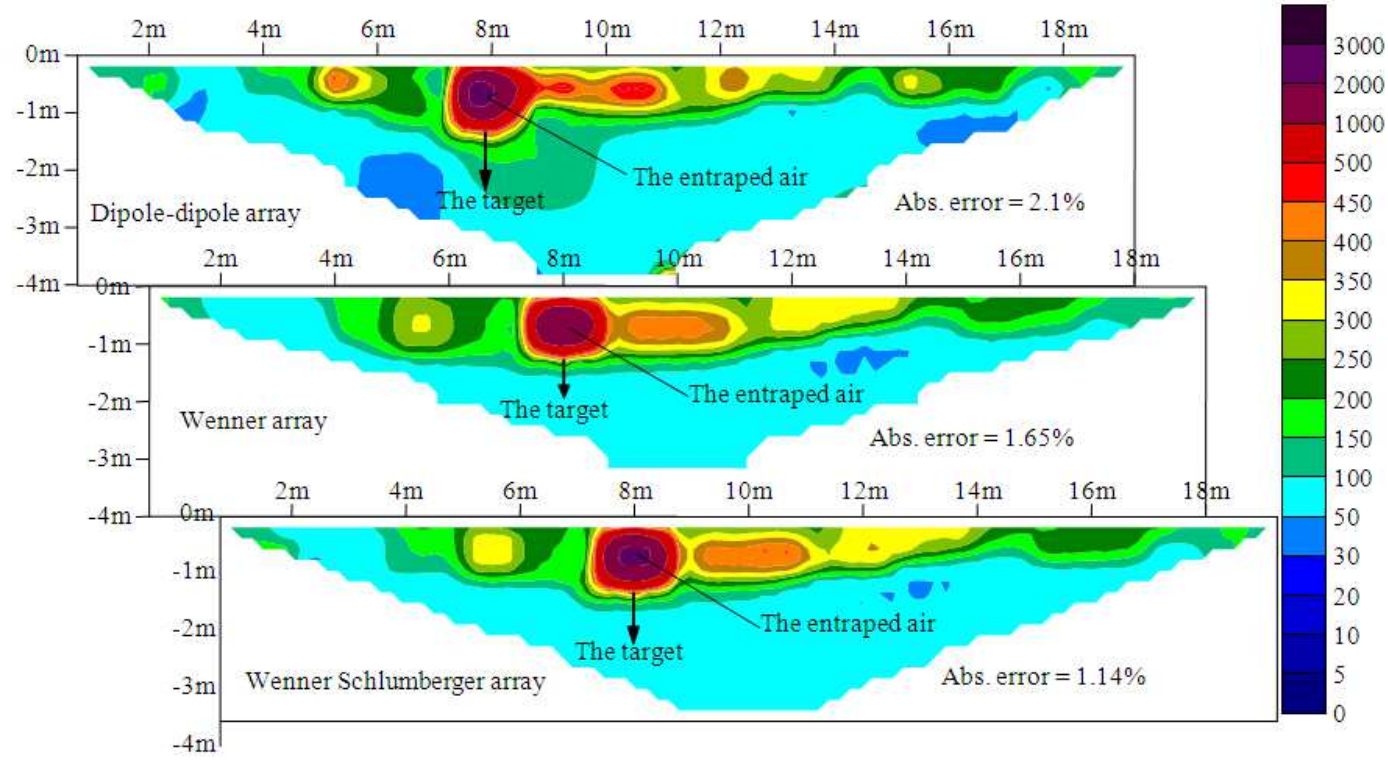

Fig. 4. Inversion results for the three Array Configurations with $0.5 \mathrm{~m}$ electrode spacing using the Robust constrain inversion technique

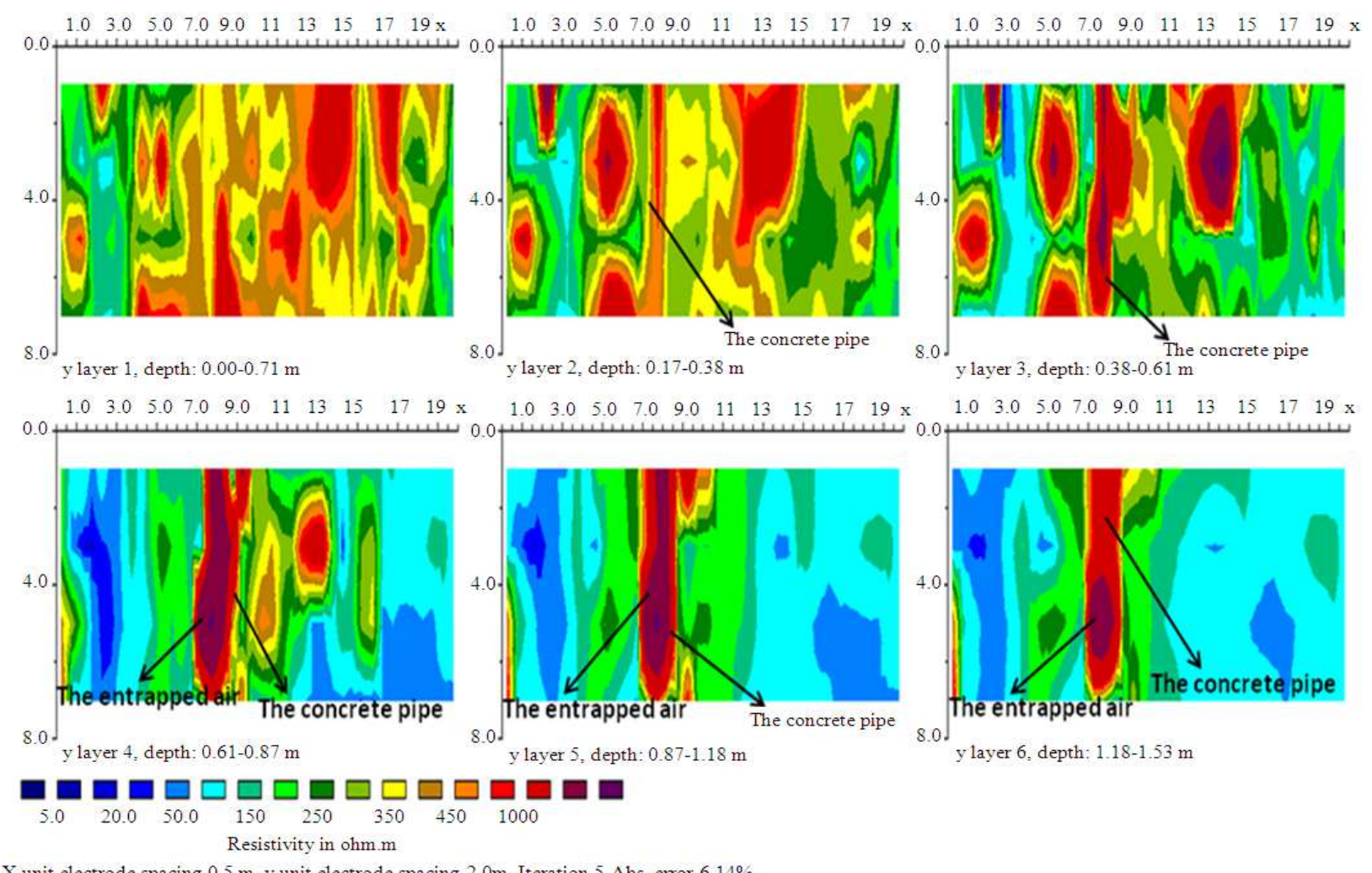

$\mathrm{X}$ unit electrode spacing $0.5 \mathrm{~m}$. $\mathrm{y}$ unit electrode spacing $2.0 \mathrm{~m}$. Iteration 5 -Abs. error $6.14 \%$

Fig. 5. 3D horizontal slices of the subsurface at various depths 


\subsection{Discussions of Results of the Robust Constrain Inversion Technique}

In a similar manner to the results of the standard constrain inversion technique, it is shown from the results of the robust constrain inversion technique shown in Fig. 3 that none of the three array configuration successfully mapped the target. The same reason given in Section 4.1 above also accounts for the inability of any of the array type to map the target.

It is obvious from Fig. 4 that all the three array configurations successfully mapped the target. In addition to this, the boundary between the entrapped air and the concrete pipe was delineated by all the three array types. This shows that the robust inversion algorithm technique gives the best boundaries resolution results. This suggests that if there is a significant contrast in the subsurface layers' resistivities, the robust constrain inversion algorithm technique gives better boundaries resolution irrespective of the array types used for the survey.

\subsection{Discussions of Results of the Inversion of the 3D Data Set}

It is clear from the results of the Inversion of the 3D data set presented in Fig. 5 that the target is well resolved. It is however shown the concrete pipe and the entrapped air are well resolved at the last three depth slices (i.e., between depths of 0.61 and $1.53 \mathrm{~m}$ ). The 3D view of the target shows that the body is elongated in a direction perpendicular to the profile direction. In addition to this, the lateral and vertical extents as well as the height of the body are clearly displayed. The result of the 3D survey has assisted in having a detail and correct perception of the subsurface target.

\section{CONCLUSION}

In this study, the effects of the choice of appropriate electrode spacing and inversion algorithms on the efficacy of $2 \mathrm{D}$ imaging to map subsurface features had been investigated. The target under investigation is the drainage concrete pipe buried at approximately $0.3 \mathrm{~m}$ into the subsurface. A profile perpendicular to the strike of the pipe was established. 2D resistivity data was separately collected with the electrode spacings of 1.5 and $0.5 \mathrm{~m}$. Three array configurations (The DipoleDipole, the Wenner and the Wenner-Schlumberger) were used for the data acquisition. The data collected was inverted using the RES2DINV program. The standard constrains and the robust constrains inversion techniques were used to do the inversion of the field data. The results obtained showed that when the electrode spacing of $1.5 \mathrm{~m}$ was used for the investigations, none of the three array types was able to map the target with either of the two inversion techniques. The results further show that the attainment of RMS error of less about $10 \%$ which usually gives the indication of a good subsurface model is not a guarantee that subsurface features are successfully mapped. On the other hand, when the electrode spacing of $0.5 \mathrm{~m}$ was used for the data collection, the results obtained with the standard constrains inversion technique showed that all the three array configurations mapped the target however, only the dipole-dipole array was able to resolve the boundary between the concrete pipe and the entrapped air. With the robust constrain inversion technique; the target was also successfully mapped by all the three array types. In addition to this, the boundary between the entrapped air and the concrete pipe was resolved by all the three array types. This shows that the robust inversion algorithm technique gives the best boundaries resolution results. This suggests that if there is a significant contrast in the subsurface layers' resistivities, the robust constrain inversion algorithm technique gives better boundaries resolution irrespective of the array types used for the survey.

In order to obtain the actual geometry of the target, a 3D survey was carried out. This was achieved by establishing additional four profiles parallel to the 2D profile earlier established. 2D data was acquired in all the profiles and the data obtained were collated into 3D format. The inversion of the collated data gave 3D resistivity sections which were presented as horizontal depth slices. The result obtained from the inversion of the $3 \mathrm{D}$ data has assisted us in getting information about the dimension and orientation of the target. This information is quite important in engineering site investigation or environmental studies.

The results obtained from this study clearly show that the importance of appropriate choice of electrode spacings and inversion algorithms for a successful mapping of subsurface features cannot be overemphasized. The study revealed that a miniature subsurface feature can only be effectively delineated if the feature is not deeply buried into the subsurface. Thus, the effectiveness of resistivity imaging to map such feature is largely limited. This study has strong implications to the applications of 2D resistivity imaging to subsurface investigations particularly in environmental studies, engineering site investigations, archaeological studies. 


\section{ACKNOWLEDGEMENT}

This project is funded by the Universiti Sains Malaysia Short Term Research Grant no. 304/PFIZIK/631005.

\section{REFERENCES}

Alfouzan, F.A., M.H. Loke and M.N.M. Nawawi, 2010. An evaluation of optimization strategies to automatically select the optimal set of array configurations for 2D electrical imaging surveys. J. Geophys. Eng., 7: 332-342. DOI: 10.1088/17422132/7/3/011

Chambers, J.E., P.B. Wilkinson, D.A. Gunn, R.D. Ogilvy and G.S. Ghataora et al., 2007. Non-invasive characterization and monitoring of earth embankments using Electrical Resistivity Tomography (ERT). Proceedings of the 9th International Conference Railway Engineering, (ICRE' 07), London.

deGroot-Hedlin, C. and S.C. Constable, 1990. Occam's inversion to generate smooth, two-dimensional models from magnetotelluric data. Geophysics, 55: 1613-1624. DOI: 10.1190/1.1442813

Friedel, S., A. Thielen and S.M. Springman, 2008. Investigation of a slope endangered by rainfallinduced landslides using 3D resistivity tomography and geotechnical testing. J. Applied Geophys., 60: 100-114. DOI: 10.1016/j.jappgeo.2006.01.001

Karl, L., T. Fechner, M. Schevenels, S. Francois and G. Degrande, 2011. Geotechnical characterization of a river dyke by surface waves. Near Surface Geophys., 9: 515-527. DOI: 10.3997/18730604.2011030

Loke, M.H. and R.D. Barker, 1996. Rapid least-squares inversion of apparent resistivity pseudosections by a quasi-Newton method. Geophysical Prospect., 44: 131-152. DOI: 10.1111/j.1365-2478.1996.tb00142.x

Loke, M.H., I. Acworth and T. Dahlin, 2003. A comparison of smooth and blocky inversion methods in 2D electrical imaging surveys. Explor. Geophys., 34: 182-187.

Loke, M.H., 2002. Tutorial: 2-D and 3-D electrical imaging surveys.
Loke, M.H., 2010. Rapid 2-D Resistivity and IP inversion using the least-squares method. Geoelectrical Imaging 2D and 3D GEOTOMO Software, Malaysia.

Martorana, R., G. Fiandaca, A.C. Ponsati and P.L. Cosentino, 2009. Comparative tests on different multi-electrode arrays using models in near-surface geophysics. J. Geophys. Eng., 6: 1-20. DOI: 10.1088/1742-2132/6/1/001

Mochales, T., A.M. Casas, E.L. Pueyo, O. Pueyo and M.T. Roman et al., 2008. Detection of underground cavities by combining gravity, magnetic and ground penetrating radar surveys: A case study from the Zaragoza area, NE Spain. Environ. Geol., 53: 1067 1077. DOI: $10.1007 / \mathrm{s} 00254-007-0733-7$

Nyari, Z. and A.I. Kanli, 2007. Imaging of buried 3D objects by using electrical profiling methods with GPR and 3D geoelectrical measurements. J. Geophys. Eng., 4: 83-93. DOI: 10.1088/17422132/4/1/010

Rybakov, M., V. Goldshmidt, L. Fleischer and Y. Rotstein, 2001. Cave detection and 4-D monitoring: A microgravity case history near the Dead Sea. Lead. Edge, 20: 896-900. DOI: 10.1190/1.1487303

Santos, F.A.M. and A.S. Sultan, 2008. On the 3-D inversion of vertical electrical soundings: Application to the South Ismailia area-Cairo desert road, Cairo, Egypt. J. Applied Geophys., 65: 97-110. DOI: $10.1016 /$ j.jappgeo.2008.06.001

Santos, F.A.M., A.R.A. Afonso and A. Dupis, 2007. 2D joint inversion of $\mathrm{dc}$ and scalar audiomagnetotelluric data in the evaluation of low enthalpy geothermal fields. J. Geophys. Eng., 4: 5362. DOI: $10.1088 / 1742-2132 / 4 / 1 / 007$

Sass, O., R. Bell and T. Glade, 2008. Comparison of GPR, 2D-resistivity and traditional techniques for the subsurface exploration of the Oschingen landslide, Swabian Alb (Germany). Geomorphology, 93: 89-103. DOI: 10.1016/j.geomorph.2006.12.019

Zhou, W., B.F. Beck and A.L. Adams, 2002. Effective electrode array in mapping karst hazards in electrical resistivity tomography. Environ. Geol., 42: 922-928. DOI: $10.1007 / \mathrm{s} 00254-002-0594-\mathrm{z}$ 